\title{
Nontraumatic Myositis Ossificans in a Muscle of Mastication
}

\author{
Tadahiko Utsunomiya1", Masaaki Suemitsu1, Miyuki Morikawa1, Masanobu Matsuno², \\ Hirotsugu Yamamoto', Hitomi Sakata', Kazuhiro Hasegawa1, Masahiro Higuchi', \\ Kayo Kuyama ${ }^{1}$ \\ ${ }^{1}$ Department of Oral Pathology, Nihon University School of Dentistry at Matsudo, Matsudo, Japan \\ ${ }^{2}$ Department of Anatomy, Nihon University School of Dentistry at Matsudo, Matsudo, Japan \\ Email: "utsunomiya.tadahiko@nihon-u.ac.jp
}

Received 30 March 2016; accepted 8 May 2016; published 11 May 2016

Copyright (C) 2016 by authors and Scientific Research Publishing Inc.

This work is licensed under the Creative Commons Attribution International License (CC BY). http://creativecommons.org/licenses/by/4.0/

(c) (i) Open Access

\begin{abstract}
Myositis ossificans (MO) is an extraskeletal tumor-like lesion with bone formation, and is relatively rare in the head and neck region. We report herein a clinicopathological analysis of MO associated with a muscle of mastication. The patient was a 70 -year-old Japanese woman who presented with a tumorous mass in the right parotid area and no history of trauma. The surgically excised tumorous lesion consisted of proliferating fibroblastic cells with no atypia in the central portion, and a formation of trabecular osteoid and/or bony tissue in the peripheral portion as the so-called "zonal phenomenon". The final histological diagnosis was MO, and the lesion was located in the posterior belly of the digastric muscle on computer tomography. A review of the literature showed that many cases of MO predominantly affected the medial pterygoid and masseter muscle and showed a history of previous trauma. The present case represents a rare case of nontraumatic $\mathrm{MO}$ in the uncommon location of the posterior belly of the digastric muscle.
\end{abstract}

\section{Keywords}

Myositis Ossificans, Muscle of Mastication, Heterotopic Ossification, Histopathology

\section{Introduction}

Myositis ossificans (MO), a kind of heterotopic ossification in muscle tissue, is a benign, reactive lesion often, but not always, associated with previous trauma [1]. MO can be subclassified into 4 subtypes: 1) MO progressiva; 2) MO traumatica; 3) MO associated with neuromuscular and chronic disease; and 4) nontraumatic MO [2].

"Corresponding author.

How to cite this paper: Utsunomiya, T., Suemitsu, M., Morikawa, M., Matsuno, M., Yamamoto, H., Sakata, H., Hasegawa, K., Higuchi, M. and Kuyama, K. (2016) Nontraumatic Myositis Ossificans in a Muscle of Mastication. Open Journal of Stomatology, 6, 135-139. http://dx.doi.org/10.4236/ojst.2016.65017 
Histopathological features typically show immature osteoid, mature bone formation and cellular fibroblastic tissues. Because the cellular fibrous and bony tissues are sometimes confused with extraskeletal osteosarcoma and other neoplastic lesions [1], recognition and differentiation of MO are important. MO predominantly affects the limbs, and is uncommon in the head and neck region. In summary reviews of the literature of the head and neck region, the major locations have been the muscles of mastication such as the masseter and pterygoid muscles, with a few cases involving neck muscles, and many cases have shown a history of trauma or dental treatment [3] [4]. We encountered an extremely rare case of nontraumatic MO in the uncommon location of the posterior belly of the digastric muscle, and reported and discussed the clinical summary and histopathological characteristics with reference to the literature.

\section{Case Presentation}

A 70-year-old woman came to Nihon University Hospital at Matsudo with a chief complaint of discomfortaround the pharynx and cough. Family history was non-contributory. The patient had experienced mastopathy and reflux esophagitis, but no trauma of the head and neck, and reported no history of pain or trismus. Computed tomography (CT) showed a tumorous mass with calcification near the right side of the first vertebra (Figure 1). The tumorous tissue was excised under a clinical diagnosis of benign parotid tumor.

The excised lesion was a solid, irregular ovoid tumor mass with calcification, measuring $4.5 \times 2.3 \times 1.5 \mathrm{~cm}$ and gray/black in coloration (Figure 2). The specimen was dehydrated in a graded ethyl alcohol series, clarified in xylene, and embedded in paraffin. The paraffin-embedded specimens (paraffin blocks) were cut into 4- $\mu \mathrm{m}$ thick sections and stained with hematoxylin and eosin (HE), Masson trichrome, and Elastica van Gieson staining (EvG).

Histopathological examination showed transitional features from proliferating fibroblastic cells at the center of the lesion to osteoid and trabecular bony tissues at the periphery, adjacent to existing muscle tissues and arteries (Figure 3(a) and Figure 3(b)). The osteoid matrix and bone were lined by osteoblastic cells. Masson trichrome staining showed blue-stained osteoid matrix, whereas mature bone matrix was reddish (Figure 3(c)). The transitional feature corresponded to the "zonal phenomenon” known as a typical feature of MO. The fibroblastic component was also intermingled with infiltrating inflammatory cells and dilated vessels (Figure 3(d)). The proliferating fibroblastic cells showed mild cellular pleomorphism with hyperchromasia and high cellularity, but no atypia or mitotic figures (Figure 3(d) and Figure 3(e)). In addition, elastic fibers, stained blackish by EvG, were observed perivascularly to the bony tissue zone (Figure 3(f)).

From these histopathological and CT findings, the final diagnosis was MO in the posterior belly of the digastric muscle.

The patient provided informed consent after the summary and purpose of the case report was explained to her.
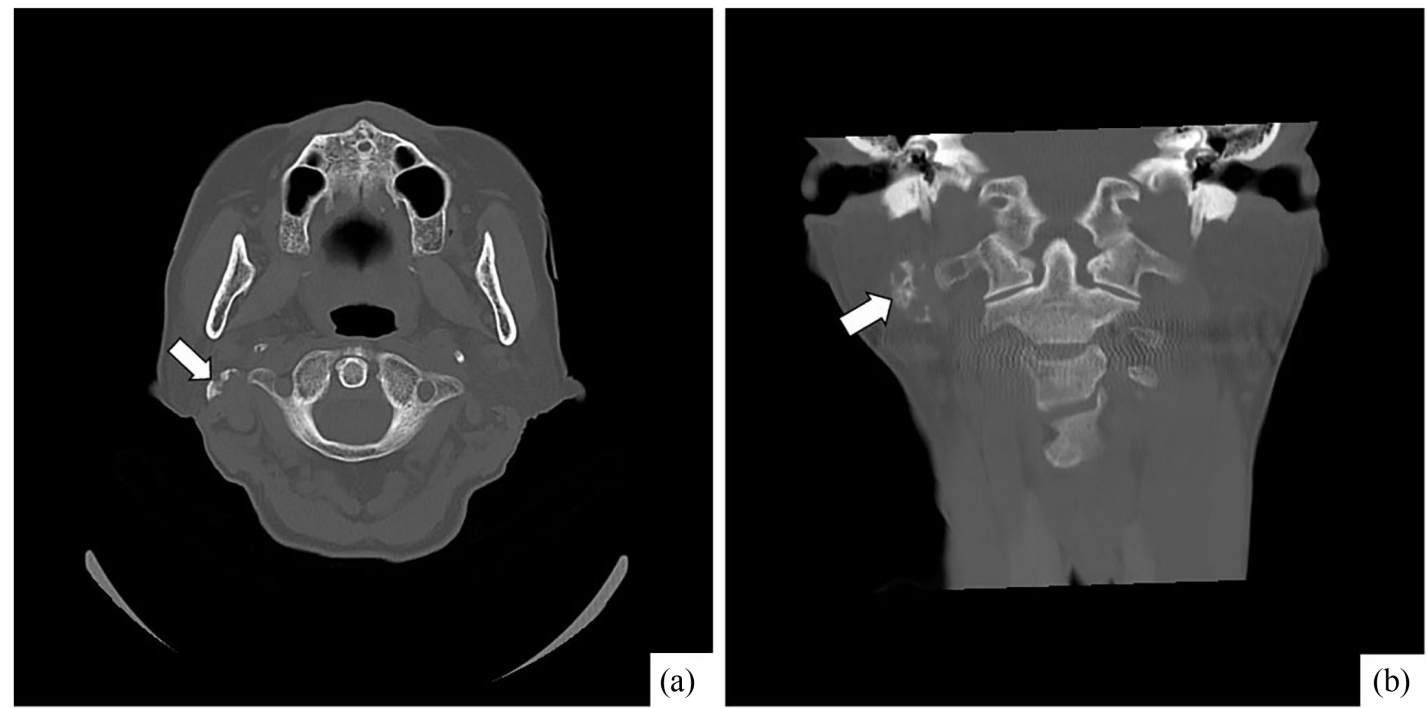

Figure 1. Computed tomography showing a calcified mass (arrow) near the right side of the first vertebra. 


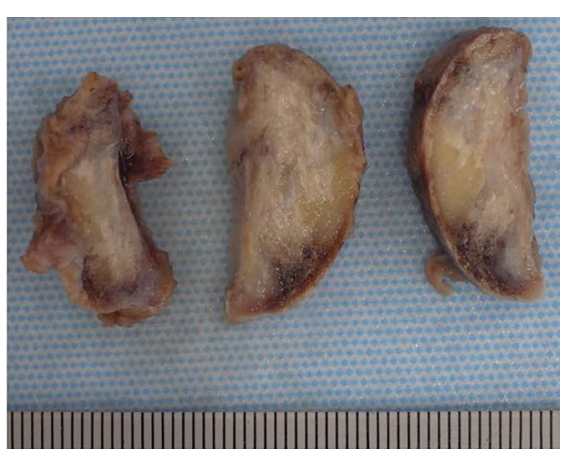

Figure 2. Cut surface of excised tissue shows a solid tumorous mass with calcification.
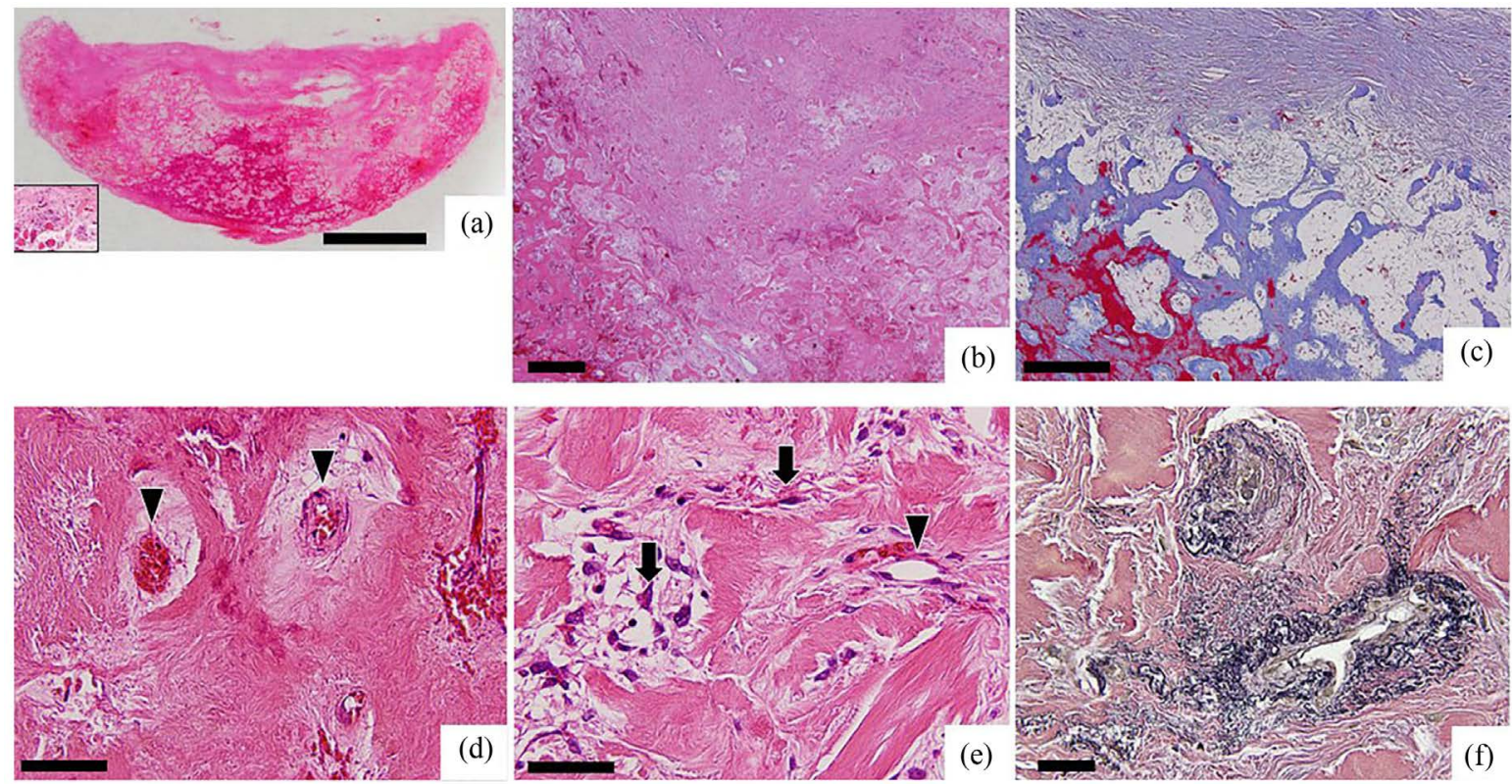

Figure 3. Histopathlogical features. (a) Gross features of the excised lesion by hematoxylin-eosin staining (inset: muscle tissue adjacent to tumorous tissues; original magnification 1×; scale bar, $10 \mathrm{~mm}$ ); (b) Low-power view showing zonal phenomenon and cellular fibroblastic component by hematoxylin-eosin staining (original magnification $20 \times$; scale bar, $500 \mu \mathrm{m}$ ); (c) Azan Mallory staining indicating transformation from fibrous tissue to osteoid (blue) and mature bone matrices (red) (original magnification 50×; scale bar, $300 \mu \mathrm{m}$ ); (d) Middle-power view showing small vessels (arrowheads) around bone formation by hematoxylin-eosin staining (original magnification 100×; scale bar, $150 \mu \mathrm{m}$ ); (e) High-power view indicating a small vessel (arrowhead) and pleomorphic mesenchymal cells (arrows) by hematoxylin-eosin staining (original magnification 300×; scale bar, $50 \mu \mathrm{m}$ ); (f) Elastica van Gieson staining showing elastic fibers stained blackish (original magnification 200×; scale bar, $50 \mu \mathrm{m})$.

\section{Discussion}

MO in the muscles of mastication and facial expression are rare, but have been summarized in detail by Jiang et al. [3] and Aoki et al. [4]. We reviewed several additional cases [5]-[17] and provided a conclusive re-summarization. Patient age ranged from the first to ninth decade of life (median, fifth decade), and a slight male predominance was recognized. Both single and multiple cases have been reported, with single cases predominating. Major sites were the medial pterygoid and masseter muscle, with a few cases involving the buccinator muscle [16] and mentalis muscle [18] [19]. Multiple MO typically involved combined occurrences in bilateral pterygoid muscles, pterygoid and masseter muscles, external pterygoid muscle, or temporalis muscle. Because of the predominance of the muscles of mastication, the major chief complaint or clinical manifestation was trismus and pain, but the present case showed non-specific symptom except for discomfort around the pharynx and cough. Atypically compared to previous cases, the present case was an elderly woman. Our review of the English lite- 
rature suggests that the present case represents the first case of a single occurrence in the posterior belly of the digastric muscle, as defined from CT. Although MO is commonly associated with previous trauma or dental treatment, cases with no such contributory history have been known as nontraumatic MO since Samuelson et al. [2] first suggested this as a fourth subtype of MO. The present case corresponded to the forth subtype from the findings of the present case compared to the reported cases.

Histopathologically, MO typically shows transitional features from cellular fibroblastic components to immature osteoid and mature trabecular bone tissues, and this feature is called the "zonal phenomenon" [1]. Transitional features were also clearly identified with bluish (uncalcified collagenous matrix) and reddish (calcified bone matrix) matrices on Masson trichrome staining. From the perspective of the differential diagnosis, MO histopathologically resembles extraskeletal osteosarcoma and other malignant tumors in soft tissue, because MO involves cellular fibroblastic components. However, osteosarcoma does not show the "zonal phenomenon", while cellular atypia is prominent. Because the present lesion showed mild cellular pleomorphism but no atypia or mitotic figures, osteosarcomas and other malignant tumors were excluded. In addition, because a cellular fibroblastic component is generally found in inflammatory pseudotumors and nodular fasciitis, this component of MO could be considered as a characteristic feature of non-neoplastic reactive lesions. MO could be differentiated from these lesions because of the presence of the zonal phenomenon.

The bone formation in $\mathrm{MO}$ is thought to be associated with heterotopic ossification or metaplasia. Although the details remain unclear, the presence of osteogenic stem cells and some cytokines and growth factors may be related to this heterotopic ossification. Morozov et al. [20] examined mesenchymal stem cells in human sarcomas and suggested that sarcoma-derived benign mesenchymal stem cells showing characteristics of pericytes are associated with differentiation into bone-forming cells. Inagaki et al. [21] recently found expression of dentine matrix protein-1 (DMP-1) in osteoblastic cells in MO, and indicated that this protein is a matrix marker of bone formation and mineralization in soft-tissue tumors. In the present case, dilated vessels were observed around the bony tissues, suggesting that the pericytes of the dilated vessels might have been involved in the osteoblastic differentiation. Bone development is generally and embryologically categorized into intramembranous and endochondral ossification. Because the MO in the present case showed osteoid and trabecular bone formation lined by osteoblastic cells, but no evidence of cartilaginous tissue, the heterotopic ossification resembled intramembranous ossification and suggested a characteristic feature in the formation of MO. Furthermore, elastic fibers were detected from the perivascular area to osteoid and bone areas with EvG. Although the biological significance of this finding is unclear, elastic fibers and their metabolism may be associated with bone matrix formation.

Because few cases of malignant transformation into parosteal osteosarcoma have been reported [2] [22], benign heterotopic ossification may be based on an osteogenic malignant pathogenesis. The most common treatment for MO is complete excision followed by physiotherapy. Furthermore, Becker et al. [5] suggested that long-term follow-up using CT is necessary, since significant calcified evidence of recurrence can occur within 12 months. Therefore, complete excision and long-term follow-up are necessary and important for nontraumatic MO to obtain well prognosis on oral surgery clinic. The present case has shown no recurrence as of 2 years after surgery.

\section{Acknowledgements}

We thank Mr. Takashi Matsumoto, CT, MT, at Division of Diagnostic Pathology, Nihon University Hospital at Matsudo for his technical support.

\section{References}

[1] Weiss, S.W. and Goldblum, J.R. (2001) Enzinger and Weiss’s Soft Tissue Tumors. 4th Edition, Mosby, $1389-1397$.

[2] Samuelson, K.M., and Coleman, S.S. (1976) Nontraumatic Myositis Ossificans in Healthy Individuals. The Journal of the American Medical Association, 235, 1132-1133. http://dx.doi.org/10.1001/jama.1976.03260370040030

[3] Jiang, Q., Chen, M.-H., Yan, C., Qiu, Y.-T., Tian, Z., Zhang, Z.-Y. and Qiu, W.-L. (2014) Post-Infectious Myositis Ossificans in Medial, Lateral Pterygoid Muscles: A Case Report and Review of the Literature. Oncology Letters, 9, 920-926. http://dx.doi.org/10.3892/ol.2014.2710

[4] Aoki, T., Naito, H., Ota, Y. and Shiki, K. (2002) Myositis Ossificans Traumatica of the Masticatory Muscles: Review of the Literatures and Report of a Case. Journal of Oral Maxillofacial Surgery, 60, 1083-1088. http://dx.doi.org/10.1053/joms.2002.34427 
[5] Becker, O.E., Avelar, R.L., Rivero, E.R.C., De Oliveira, R.B., Meurer, M.I., Santos, A.M.B., Júnior Haas, O.L. and Meurer, E. (2015) Myositis Ossificans of the Temporalis Muscle. Head and Neck Pathology, Online, 24. http://dx.doi.org/10.1007/s12105-015-0675-4

[6] Torres, A.M., Nardis, A.C., da Silva, R.A. and Savioli, C. (2015) Myositis Ossificans Traumatica of the Medial Pterygoid Muscle Following a Third Molar. International Journal of Oral Maxillofacial Surgery, 44, 488-490. http://dx.doi.org/10.1016/j.ijom.2014.11.003

[7] Demikol, M., Aras, M.H. and Tutar, E. (2015) Myositis Ossificans Circumscripta in the Masseter Muscle Mimicking Phleboliths. Journal of Carniofacial Surgery, 26, 2020-2021. http://dx.doi.org/10.1097/SCS.0000000000001897

[8] Kumar, N., Austin, R.D., Mathew, P., Sakthivel, S. and Vijayalakshmi, L. (2014) Traumatic Myositis Ossificans of the Masseter Muscle: A Case Report with Conventional and Advanced Imaging Features. General Dentistry, 62, 75-77.

[9] Almeida L.E., Doetzer, A., Camejo, F. and Bosio, J. (2014) Operative Management of Idiophatic Myositis Ossificans of Lateral Pterygoid Muscle. International Journal of Surgical Case Reports, 5, 796-799. http://dx.doi.org/10.1016/j.ijscr.2014.09.008

[10] Reddy, S.P.D., Prakash, A.P. and Rao, B. (2014) Myositis Ossificans Traumatica of Temporalis and Medial Pterygoid muscle. Journal of Oral Maxillofacial Pathology, 18, 271-275. http://dx.doi.org/10.4103/0973-029X.140781

[11] Spinzia, A., Moscato, G., Broccardo, E., Castelletti, L., Maglitto, F., Orbana, G.D. and Piombino, P (2014) A Rare Isolated Unilateral Myositis Ossificans Traumatica of the Lateral Pterygoid Muscle: A Case Report. Journal Medical Case Reports, 8, Online. http://dx.doi.org/10.1186/1752-1947-8-230

[12] Kamalapur, M.G., Patil, P.B., Joshi, S. and Shastri, D. (2014) Pseudomalignant Myositis Ossificansinvoving Multiple Masticatory Muscles: Imaging Evaluation. Indian Journal of Radiology and Imaging, 24, 75-59. http://dx.doi.org/10.4103/0971-3026.130706

[13] Baliga, M. and Baptist, J. (2014) Myositis Ossificans of Infraorbital Musculature in Uncontrolled Diabetic. New York State Dental Journal, 80, 47-49.

[14] Piombino, P., Orbana, G.D., Abbate, V., Fini, G., Limeratore, G.M., Micl, E. and Belli, E. (2013) Circumscribed Myositis Ossificans of the Masseter Muscle: Report of a Case. Il Giornale di Chirurgia, 34, 271-274.

[15] Schiff, M.J. and Meara, D.J. (2013) Myositis Ossificans of the Temporalis Muscle: Case Report and Review of the Literature. Journal of Oral Maxillofacial Surgery, 71, 1893-1898. http://dx.doi.org/10.1016/j.joms.2013.05.002

[16] Deliverska, E.G. (2013) Myositis Ossificans Traumatica of the Masseter Muscle. Review of the Literature and Case Report, 19, 411-414.

[17] Wiggins, R.L., Thurber, D.T., Abramovitch, K., Bouquot, J. and Vigneswaran, N. (2008) Myositis Ossificans Circumscripta of the Buccinator Muscle: First Report of a Rare Complication of Mandibular Third Molar Extraction. Journal of Oral Maxillofacial Surgery, 66, 1959-1963. http://dx.doi.org/10.1016/j.joms.2008.01.066

[18] Johansson, B. (1984) Traumatic Myositis Ossificans. Oral Surgery Oral Medicine Oral Pathology, 58, 742. http://dx.doi.org/10.1016/0030-4220(84)90046-X

[19] Mulherin, D. and Schow, C.E. (1980) Traumatic Myositis Ossificans after Geniplasty. Journal of Oral Surgery, 38, 786-789.

[20] Morozov, A., Downey, R.J., Healey, J., Moreira, A.L., Franceschino, A., Dogan, Y., Leung, R., Edgar, M., LaQuaglia, M., Maki, R.G. and Moore, M.A.S. (2010) Benign Mesenchymal Stromal Cells Human Sarcomas. Clinical Cancer Research, 16, 5630-5640. http://dx.doi.org/10.1158/1078-0432.CCR-09-2886

[21] Inagaki, Y., Kashima, T.G., Hookway, E.S., Tanaka, Y., Hassan, A.B., Oppermann, U. and Athanasou, N.A. (2015) Dentine Matrix Protein 1 (DMP-1) Is a Marker of Bone Formation and Mineralisation in Soft Tissue Tumours. VirchowsArchiv, 466, 445-452. http://dx.doi.org/10.1007/s00428-014-1706-3

[22] Wheeler, K., Markary, R. and Berrey, H. (2014) A Case of Malignant Transformation of Myositis Ossificans. American Journal of Orthopedics, 43, E25-E27. 